\title{
Dust and Metallicity across local Galaxies
}

\author{
Brent Groves \\ Research School of Astronomy \& Astrophysics, Australian National University, \\ Mt. Stromlo Observatory, Cotter Rd, Weston Creek, ACT, Australia \\ email: brent.groves@anu.edu.au
}

\begin{abstract}
The gas-phase metallicity is one of the key insights we have into measuring the chemical feedback of stars in galaxies, yet remains difficult to correctly determine. However, there is a close relation between gas-phase metals and dust in the ISM, which can give us insight into the correct metallicity in galaxies. Here I will present recent work on metallicity gradients in galaxies, and how we can measure the global metallicity and the radial gradients in nearby galaxies using dust, through the dust-to-gas ratio.
\end{abstract}

Keywords. ISM: Dust, ISM:abundances, Galaxies

\section{Introduction}

The abundance of metals in the gas phase of galaxies is a measure of the chemical feedback history from stars. Thus the global metallicity and radial metallicity gradients can provide key insights into the processes of stellar evolution and galaxy growth. Measuring gas-phase abundances is typically done through emission lines requiring HII regions and star-forming galaxies.

While global metallicities have been determined through fibre spectra (e.g., SDSS; Tremonti et al. 2003), metallicity gradients have been limited to slit spectra or multiple observations of nearby galaxies until recently (e.g. Moustakas et al. 2010). Integral Field Spectra are perfect for the determination of radial abundance gradients and even smaller scale variations. Recent work based on integral field studies suggests that gas-phase abundances gradients are similar across non-interacting star-forming galaxies but only once normalised to the stellar effective radii (Sanchez et al. 2014, Ho et al. 2015).

However, one outstanding issue is that the determination of the metallicity depends critically on the measurement of the gas temperature and ionisation corrections. These can be difficult to determine, and typically astronomers rely on empirical or model-based calibrations. These different calibrations have large systematics between them (see e.g. Kewley \& Ellison 2008).

One way around this is to use the IR emission from dust. Dust can give strong insight into total metals via the dust-to-gas ratio. Dust emission also gives insights into the stellar radiation field, which can arise from both young and old stars. Nearby galaxies are the best case to do resolved comparisons of stars, gas and dust to determine both the absolute calibration and the relative calibrations.

\section{Dust in nearby galaxies}

The Andromeda galaxy (M31) is a one of a kind laboratory for stars and ISM. Its proximity mean that we can resolve individual stars with HST, and the low angular resolution IR observations can see several scales. we have mapped the dust heating and column using Herschel observations (Groves et al. 2012, Draine et al. 2014), and find consistent metal gradient with existing emission line determinations, in the inner $15 \mathrm{kpc}$, 


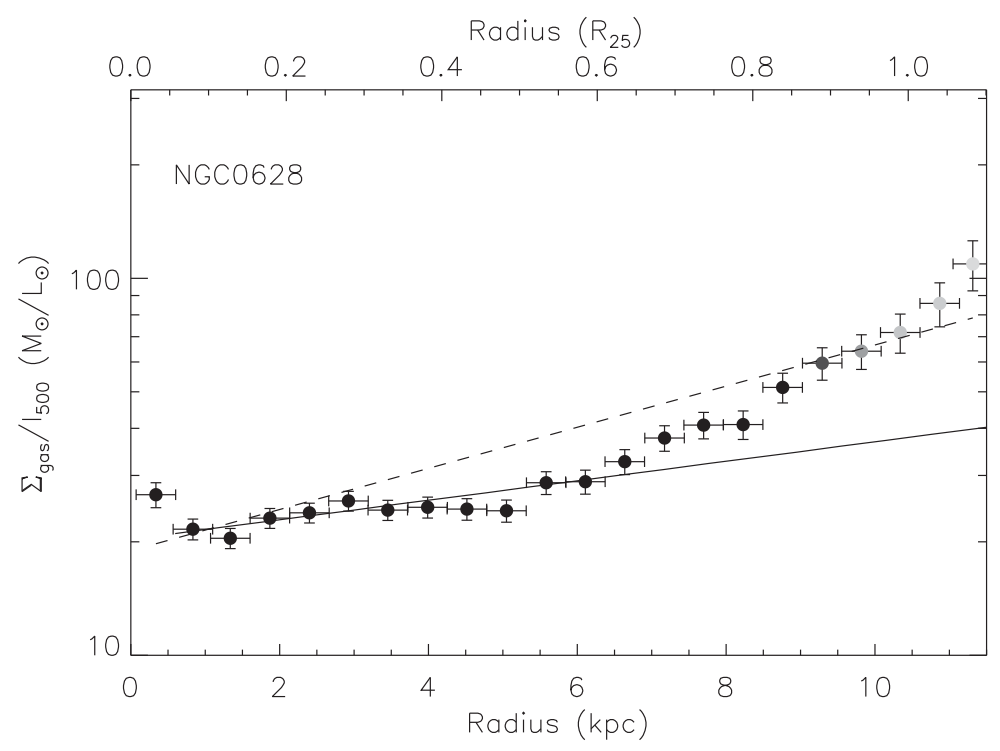

Figure 1. The gas-to-dust ratio $\left(\Sigma_{\mathrm{HI}+\mathrm{H} 2} / I_{500 \mu m}\right)$ as a function of radius in the KINGFISH galaxy NGC 628. Overlaid are the determined metallicity gradients (inverted) from Moustakas et al. 2010 from two different calibrations. This figure demonstrates both the similarity in gradients from both methods, as well as the larger extent of the dust method, and an indication of a break around $R_{25}$. Figure from Groves et al. 2015.

yet can trace the gradient out to larger distances, and see a break out at $\sim 20 \mathrm{kpc}$. Importantly with HST data we can determine star formation histories across M31 (Lewis et al. 2015) which can be matched with our measured metallicity gradient.

Using the KINGFISH sample of Herschel observed galaxies (Kennicutt et al. 2015) we extended this work to a larger sample of nearby galaxies, and found similar gradients in dust-to-gas ratios as previously determined metallicity gradients (Groves et al. 2015). We also saw a break in the dust-to-gas ratio (and therefore the metallicity gradient) close to the optical radius $\left(R_{25}\right)$, similar to what was seen in M31 (see Figure 1).

\section{Summary}

While the global gas-phase abundances scale with stellar metallicity and therefore the star formation history, the radial gradient normalised to $R_{25}$ appears to be constant. Dust can trace the global heating and the gas-phase metallicity (relative to gas) where HII regions can not. Using dust, we find the same metallicity radial gradients with respect to $R_{25}$ in nearby galaxies as gas-phase abundances, however we see indications of breaks in these gradients around the optical radius

\section{References}

Draine, B. T., Aniano, G., Krause, O., et al. 2014, ApJ, 780, 172

Groves, B., Krause, O., Sandstrom, K., et al. 2012, MNRAS, 426, 892

Groves, B. A., Schinnerer, E., Leroy, A., et al. 2015, ApJ, 799, 96

Ho, I.-T., Kudritzki, R.-P., Kewley, L. J., et al. 2015, MNRAS, 448, 2030

Kennicutt, R. C., Calzetti, D., Aniano, G., et al. 2011, PASP, 123, 1347

Kewley, L. J. \& Ellison, S. L. 2008, ApJ, 681, 1183

Lewis, A. R., Dolphin, A. E., Dalcanton, J. J., et al. 2015, ApJ, 805, 183

Sánchez, S. F., Rosales-Ortega, F. F., Iglesias-Páramo, J., et al. 2014, A\&\&A, 563, A49 